\title{
Study on the Simulation of the Soft Start of the Asynchronous Motor with Pump Control Function Based on Thyristor
}

\author{
Tingjian Zhong, Li Zhu \& Minghua Zhou \\ Jiangxi Vocational \& Technical College of Electricity, Nanchang 330032, China \\ E-mail: jxdlztj@163.com \\ Yongtao Dai \\ School of Information Engineering, Nanchang University, Nanchang 330029, China \\ E-mail: dytnc2007@126.com
}

\begin{abstract}
To reduce the impact of the high current on the power grid from the start process of the asynchronous motor and eliminate the negative influences of traditional reduced voltage start on the electronic and mechanic equipments and enhance the start character of the motor, we studied and improved the soft start of the motor, and put forward the soft starter with the function of pump control which could reduce the water hammers induced in the start and stop of the pump, reduce the oscillation of the pipeline system and perfect the functions of the soft starter. In the article, we used MATLAB to establish simulation module of the three phase current voltage regulation circuit system, and tested the soft starter designed in the article, and the simulation result indicated that the soft starter with pump control function could not only effectively reduce the starting impact of the motor to the power grid, but reduce the impact from the water hammer and optimize the start performance of the motor.
\end{abstract}

Keywords: Soft starter, Pump control, Torque, Thyristor

\section{Introduction}

The traditional start mode of motor can be divided into direct start and reduced voltage start which can be divided into Y- $\Delta$ start, auto-coupling reduced voltage start, stator string reactor start and so on, and because traditional start modes are hard to adjust the motor parameters exactly to fulfill the smooth start according to certain requirement in the start process, and induce certain impact to the power grid (Li, 2006, 185-187 \& Zhang, 1999, P. 3-5), we begin to study the soft start of the asynchronous motor. The general soft starters all have the voltage ramp start mode and limited current start mode (Xu, 2001, P. 41-44), and the soft starter designed in the article takes the thyristor as the execution component, and emits PWM wave to control the thyristor to trigger the pulse by the control unit, and realize the control of the motor start and control the connection of the thyristor. The voltage regulation circuit of thyristor has very important meaning for the implementation of the functions of the soft starter, and we mainly utilize the voltage regulation principle and voltage regulation circuit of the thyristor to study the characteristics of the soft start.

\section{Mathematical model of asynchronous motor}

To study the relationships among various variables such as the voltage, current and torque in the start and stop of the asynchronous motor, we give the mathematical model of the asynchronous motor here. When we study the frequency conversion speed regulation, we mainly adopt the mathematical model based on the state equation, and for the soft start, the mathematical model of the asynchronous motor based on centralized parameters equivalent circuit is generally adopted (Wang, 1990 \& Gao, 1993) (seen in Figure 1), where, $U_{1}$ is the phase voltage virtual value of the power grid, $r_{m}$ and $x_{m}$ respectively are the excitation resistance and the excitation reactance, $r_{1}$ and $x_{1 \sigma}$ respectively are the resistance and the leakage reactance of the stator, $r_{2}^{\prime}$ and $x_{2 \sigma}^{\prime}$ respectively are the resistance reduced value and the leakage reactance reduced value of the rotor, and $s$ is the slip ratio.

From the principle of the motor theory, the mechanical character equation expression of three phase asynchronous motor is 


$$
T_{e m}=\frac{P_{e m}}{\Omega_{1}}=\frac{3 p U_{1}^{2} \frac{r_{2}^{\prime}}{S}}{2 \pi f_{1}\left[\left(r_{1}+\frac{r_{2}^{\prime}}{S}\right)^{2}+\left(x_{1 \sigma}+x_{2 \sigma}^{\prime}\right)^{2}\right]}
$$

When the motor just starts, the rev of rotor is $n_{2}=0$, and the slip ratio is $s=1$, and the mechanical character equation of the motor here is

$$
T_{s t}=\frac{3 p U_{1}^{2} r_{2}^{\prime}}{2 \pi f_{1}\left[\left(r_{1}+r_{2}^{\prime}\right)^{2}+\left(x_{1 \sigma}+x_{2 \sigma}^{\prime}\right)^{2}\right]}
$$

Because $\left|\left(r_{1}+r_{2}^{\prime}\right)+j\left(x_{1 \sigma}+x_{2 \sigma}^{\prime}\right)\right|$ is much less than $\left|\left(r_{1}+r_{m}\right)+j\left(x_{1 \sigma}+x_{m}\right)\right|$, so when the motor is started,

$$
\begin{aligned}
& Z_{k} \approx \sqrt{\left(r_{1}+r_{2}^{\prime}\right)^{2}+\left(x_{1 \sigma}+x_{2 \sigma}^{\prime}\right)^{2}} \\
& I_{1} \approx \frac{U_{1}}{Z_{k}}
\end{aligned}
$$

Because the currents of the rotor and the stator are much bigger than the rated currents when starting the motor, the leakage reactance of the rotor and the stator makes the iron magnetic part in the leakage magnetic circuit saturated, and the leakage magnetic resistance becomes bigger, which makes $x_{1 \sigma}$ and $x_{2 \sigma}^{\prime}$ diminished, $Z_{k}$ diminished further, and the current higher. From formula (2) and formula (4), the start current is proportional to the terminal voltage, and the start torque is proportional to the square of the stator terminal voltage. When the start voltage is low, the start torque and the current are low, and if the voltage is high, the start torque and the impact current are high. If the asynchronous motor adopts the direct start mode, the voltage break from zero to the voltage of the power grid will produce large impact torque and impact current which will bring many negative influences to the electronic equipments and mechanical equipments.

\section{Principle of pump control soft start}

Through the analysis of the traditional start, the voltage ramp start (Li, 2000, P. 16-19) and the limited current start (Zenginobuz, 2000, P.1593-1604 \& Sen P, 1900, P.1102-1107), there is a common character in the general reduced voltage start, i.e. they all can not exactly adjust the motor parameters to adapt special start requirements. Though the initial torque and the initial current are reduced, but if the start is required to be implemented in the constant accelerating torque and below the special value of the current, the reduced voltage start can not fulfill these requirements. And for this start mode, the maintenance is complex, and the installation charge is high, and it can not completely avoid the current impact and torque impact, and the apparatus in the power grid may be damaged. So we put forward the pump control soft start which takes the thyristor as the execution component, and emits PWM wave to control the thyristor to trigger the pulse by the control unit, and realizes the control of the motor start and control the connection of the thyristor (Lin, 2002 \& Wang, 2001). The closed loop control frame chart is seen in Figure 2.

The pump control is actually a sort of torque control, and it adopts the PID control to adjust the electromagnetic torque (Wang, 1998 \& Zhang, 2005, P. 19-21), and it requires that the electromagnetic torque should ascend according to the pump character curve when the motor starts, i.e. the accelerating torque should be tried to keep in a stable range, and the value should not be too high, and the electromagnetic torque just exceeds the load torque. The torque/rev figure of the soft starter with pump control function is seen in Figure 3, and to comparing with the general voltage ramp start and the limited current start, we also give the torque character curves of the direct start and the voltage ramp start. We can compare the torque/rev curves of the direct start, the electric reduced voltage star and the special pump control soft start in the figure, and from the figure, we can see that after the pump control function is used, a small difference value will exist between the motor torque and the torque of pump load, which will largely reduce the accelerating torque in the start and perfectly control the output torque.

\section{Simulation research}

We used the Simulink base and SimPowerSystems in MATLAB6.5 to establish the soft starter simulation model of the asynchronous motor pump control start mode. As seen in Figure 4, the simulation module of the pump control soft starter is mainly composed by the three phase voltage power module, the triggering pulse module, the thyristor module, the measurement module and the control module. The three-AC voltage power module is connected in start by three single phase $\mathrm{AC}$ voltage supplies, and the relationship between the phase and the amplitude is set up correctly. The peak amplitudes of phase A, phase $\mathrm{B}$ and phase $\mathrm{C}$ are all $312 \mathrm{~V}$, and the voltage frequencies are all $50 \mathrm{~Hz}$, and the phases respectively are $0^{\circ}, 120^{\circ}$ and $240^{\circ}$, and the trigger circuit is composed by the synchronization stage, sawtooth wave 
forming stage and the phase-shift control stage, and the input port of the circuit is the input port of the synchronous voltage, and the synchronous voltage forms the square wave with the width of the half circle of the synchronization voltage after passing the relay stage, and the square wave produces the sawtooth wave by the rate limiter, and the sawtooth wave folds with the phase shift control voltage (input port In2) and adjusts the zero-pass point of the sawtooth wave, and after passing Relay 1, the thyristor emitting pulses which front part can be adjusted and which back part is fixed are produced, and the parameter setting of various modules is seen in Table 1.

The waveforms including the synchronization signal, half-cycle and equal width square wave, sawtooth wave, folded phase shift control and the trigger signal in turn of the various branch modules in the trigger circuit is seen in Figure 5. The thyristor module is composed by three trigger angle control modules, and it produces six ways trigger pulses, and each trigger pulse differs in $60^{\circ}$, and the simulation waveform is seen in Figure 6 . And the thyristor module is the core of the main loop of the soft starter, and it is the AC voltage regulation circuit composed by three groups including 6 thyristors.

Because the system is mainly controlled by the pump load, so the objective of the simulation is not generally constant load, but the pump load which load torque changes with the rev. The load torque of the pump load is proportional with the square of the rev, so a pump character curve of the pump load can be approximately fitted (seen in Figure 7). The current waveform and the torque waveform of the pump control start mode are seen in Figure 8 and Figure 9, and from the figures, we can clearly see that the pump control mode can not only further limit the start current (about 30A), but better control the output torque of the motor (the maximum torque is less than $55 \mathrm{~N} \cdot \mathrm{m}$ ), and the electromagnetic torque and the load torque keep simultaneously ascending, which can largely reduce the acceleration torque and the hammer impact.

\section{Conclusions}

From the simulation result, the soft start with pump control could reduce the accelerating torque and the start current to the largest extent, and its performance is much better than general starter, and it could better control the start process of the asynchronous motor.

\section{References}

Gao, Jingde, Wang, Xiangxian \& Li, Fahai. (1993). AC Motor and Its System Analysis. Beijing: Tsinghua University Press.

Li, Guohou \& Gao, Shuping. (2006). Soft Starting Technology and Its Application. Coal Mine Machinery. No.7. P. 185-187.

Lin, Weixun. (2002). Modern Power Electronics Circuit. Hangzhou: Zhejiang University Press.

Li, Wujiu. (2000). Intelligent Soft Starter. Low Voltage Apparatus. No.1. P. 16-19.

Sen P. Landa. (1900). Derating of Induction Motors Due to waveform Distoration. IEEE Trans. On Industry Application. No.11. Vol.26(3). P.1102-1107.

Wang, Yudong. (1990). Motor Theory. Hangzhou: Zhejiang University Press.

Wang, Zhao'an \& Huang, Jun. (2001). Power Electronics Technology. China Machine Press.

Wang, Zhao'an, Yangjun \& Liu, Jinjun. (1998). Harmonic Suppression and Reactive Power Compensation. China Machine Press.

Xu, Liaosong, Qian, Cunyuan \& Xie, Weida. (2001). A Soft-stop Control Method of Asynchronous Motor Soft-starter. Power Electronics. No.35(3). P. 41-44.

Zenginobuz, G. Cedircil, Ermis, M \& Barlark. C. (2000). Soft starting of large induction motors at constant current with minimized starting torque pulsations. Conference Re Cordofthe2000 IEEE. No.3(11). P.1593-1604.

Zhang, Xiaoquan. (1999). New Concept of Motor Control Protection: the Characteristics and Application Technology of the Super Soft Start Equipment. Electric Power Construction. No.8. P. 3-5.

Zhang, Xueping. (2005). Application of Single-chip Microcomputer in Soft Start of AC Motor. Industry and Mine Automation. No.4. P. 19-21. 
Table 1. Parameters setting of the triggering angle control module

\begin{tabular}{|c|c|c|c|c|c|c|c|}
\hline \multirow{2}{*}{ Module } & $\begin{array}{c}\text { Power } \\
\text { Uin }\end{array}$ & \multicolumn{2}{|c|}{ Relay1, Relay3 } & \multicolumn{2}{c|}{ Rate Limiter1, Rate Limiter2 } & \multicolumn{2}{c|}{ Relay2, Relay4 } \\
\hline \multirow{3}{*}{$\begin{array}{c}\text { Parameter } \\
\text { setting }\end{array}$} & $220 \mathrm{~V}$ & Switch On point & eps & Rising slew rate & 1000 & Switch On point & eps \\
\cline { 2 - 9 } & $50 \mathrm{~Hz}$ & Switch Off point & eps & Falling slew rate & $-1 \mathrm{e} 8$ & Switch Off point & eps \\
\cline { 2 - 9 } & & Output when on & 10 & & & Output when on & 1 \\
\hline
\end{tabular}

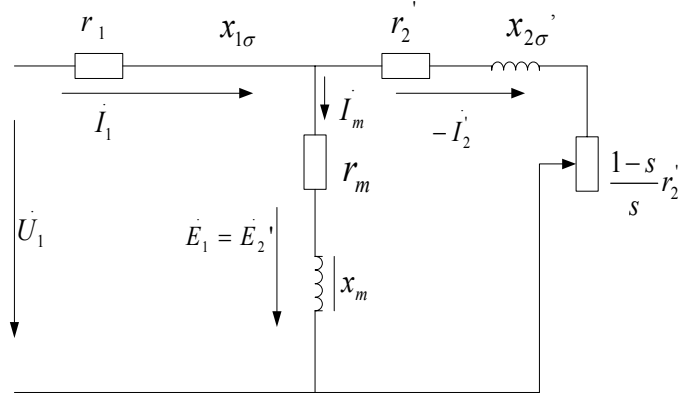

Figure 1. Single Phase Equivalent Circuit of Asynchronous Motor

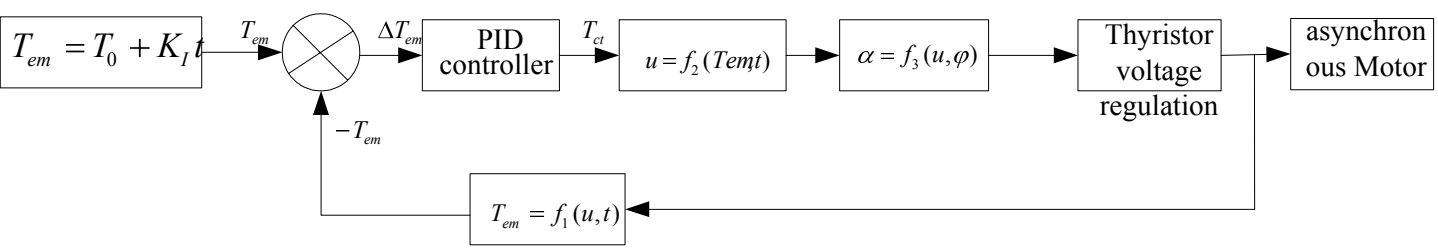

Figure 2. Torque Closed Loop Control Strategy

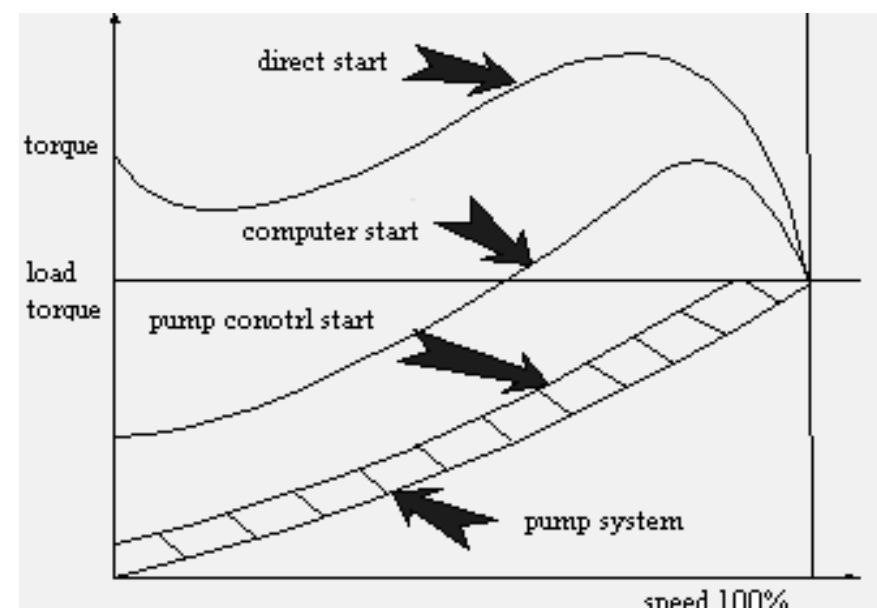

Figure 3. Torque/Rev Curve of Pump Control Soft Starter 


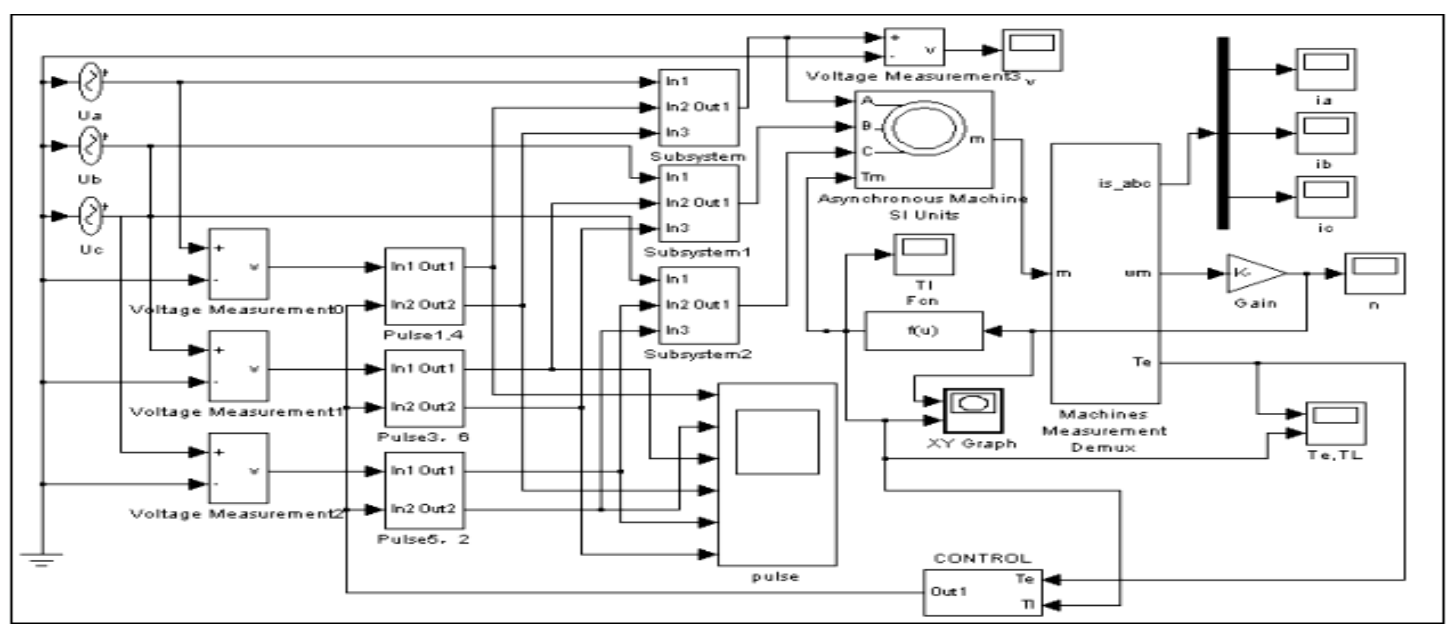

Figure 4. Simulation Principle

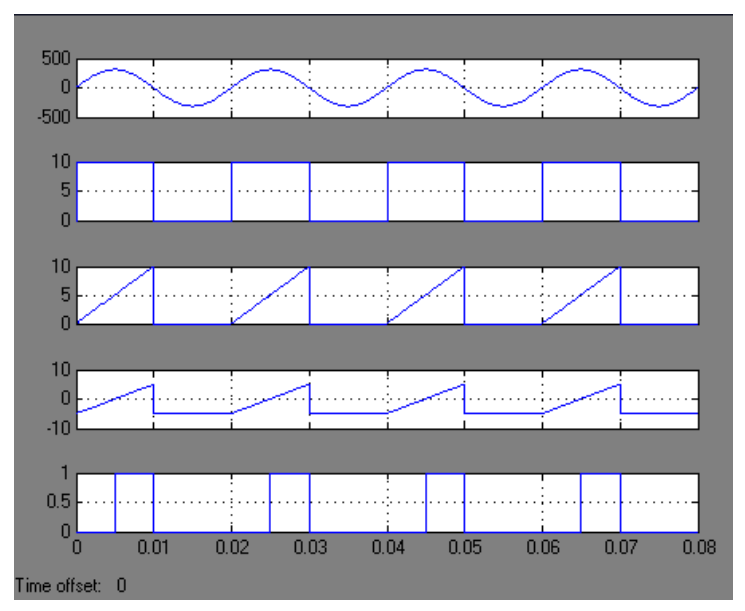

Figure 5. Various Branch Module Waveforms of Trigger Circuit

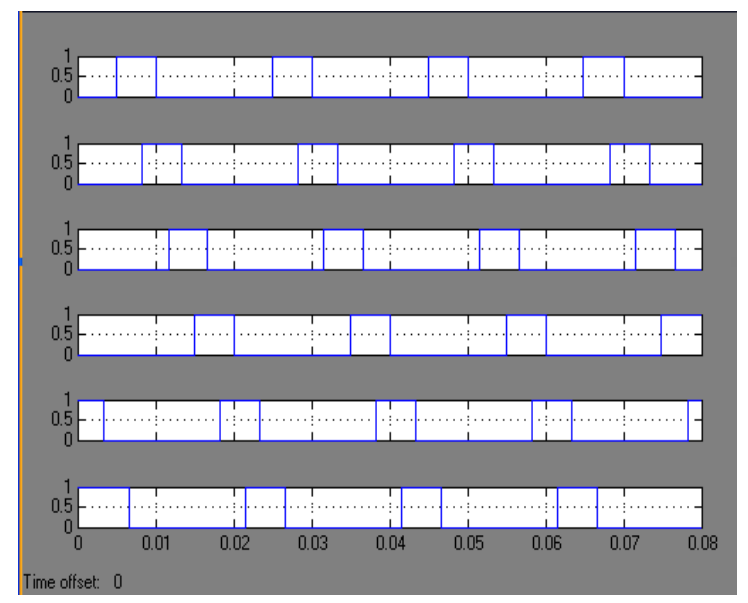

Figure 6. Six-way Pulse Outputs 


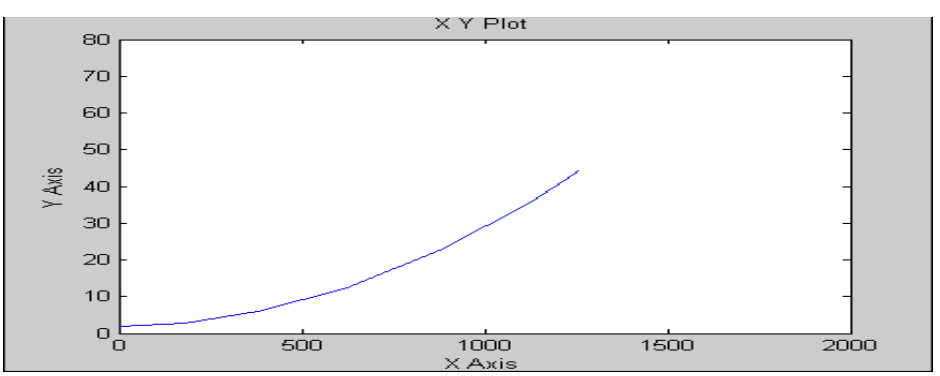

Figure 7. Speed/Torque Curve of Pump Load

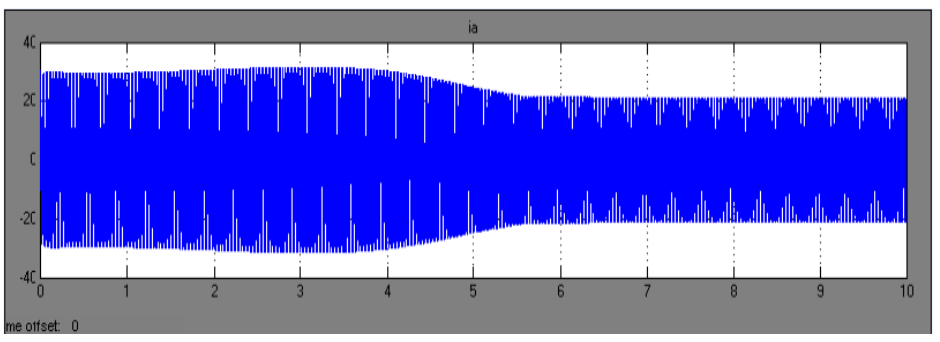

Figure 8. Pump Control Start Circuit $i_{a}$

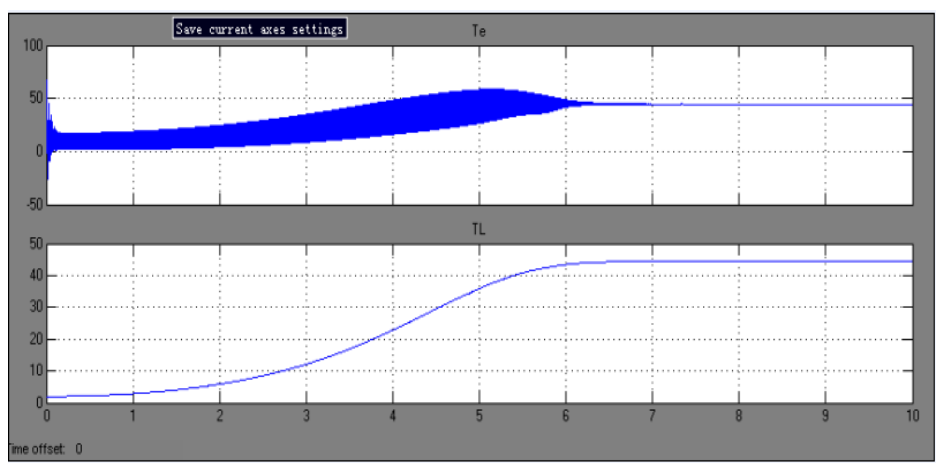

Figure 9. Pump Control Start $T_{e}, T_{L}$ 